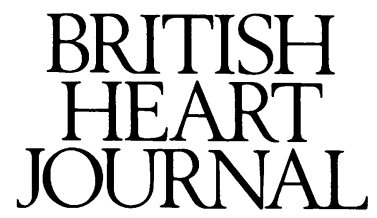

\title{
Editorial
}

\section{Alcohol catheter ablation of atrioventricular conduction}

The control of arrhythmia by catheter ablation was one of the highlights of clinical electrophysiology in the 1980s. Electrical catheter ablation of normal atrioventricular conduction has become an established technique for the creation of complete heart block in patients with atrial fibrillation and flutter uncontrolled by drugs, and has all but replaced the use of surgically created heart block. This technique lacks elegance because it does not cure the arrhythmia, but replaces it with a rhythm disturbance, heart block, that can be managed effectively with a pacemaker. It follows that the relative simplicity of this technique should not lead to its indiscriminate use in large numbers of patients, as most can be managed satisfactorily with antiarrhythmic drugs or may require no treatment. Electrical ablation is not without complications, and recent modifications have sought to improve the safety and efficacy.

While it seems logical to use electrical energy to destroy electrical function, there are other methods of damaging conducting tissue. On page 143 of this issue Sneddon et al present their experience with the new technique of transcoronary ablation of atrioventricular conduction with alcohol. They attempted ablation in 14 patients and were able to visualise and cannulate the appropriate artery in 10 $(70 \%)$. After alcohol injection, chronic complete heart block was created in six of these patients, and one further patient had persistent conduction but remains symptom free without drug treatment. The modest success rate of $43 \%$ on an intention to treat basis, the potential for serious complications, and the significant increase in cardiac enzymes lead Sneddon et al to conclude that this technique should probably be reserved for those in whom other ablative procedures have failed. Alcohol ablation was successful in one of the three patients in whom electrical ablation had failed.

Such results stimulate not only a critical evaluation of the new technique and its place alongside other techniques, but also an appraisal of more established methods. Certainly, the conventional method of ablation, by high energy defibrillator pulses and pacing catheters, has been shown to be reasonably effective ( $64 \%$ complete heart block) in a large series of patients, with low complication rates. ${ }^{1}$ In a more recent report the success rate was $88 \%$ but the incidence of serious complications was unacceptably high, especially the in-hospital mortality of $5 \%$ related to the procedure. $^{2}$

Research into alternative methods has brought two new techniques to the fore. Low energy capacitive discharges were very successful $(79-100 \%)$ and did not have the complications of high energy ablation ${ }^{3-5}$; this technique is indicated when the presenting arrhythmia is atrial fibrillation or flutter. Though radiofrequency ablation has not yet proved as effective for creating chronic complete heart block ( $36 \%$ success in the most recent series), ${ }^{2}$ it has proved very successful in modifying atrioventricular conduction in patients with atrioventricular nodal reentrant tachycardia (42-100\%) ${ }^{6-8}$ However, despite the obvious attractions of modifying atrioventricular conduction and thereby producing a more controlled ventricular rate in atrial fibrillation or flutter while avoiding the necessity for permanent pacing, there is no evidence that such an approach improves symptoms. Therefore, we think that in patients with atrial fibrillation and flutter creation of complete heart block with low energy ablation continues to be the procedure of choice.

Given the high success rates of conventional techniques, do we need another method for non-surgical ablation of atrioventricular conduction? The answer must be that, unless the new technique guarantees success to offset its possibly greater associated risks, it is unlikely to be useful.

The use of transcoronary alcohol injection to create complete heart block deserved attention, and Sneddon et al have carefully assessed its benefits and risks. Although in its present form it is disappointing, other than as an alternative when other techniques have failed, the principles of the technique deserve further research. The ability to create transient complete heart block in each case with cold saline shows that the conduction system can be reached through the coronary arteries. Could toxic substances with a highly specific affinity for conducting tissue be developed? Improvement in catheter and radiological techniques holds promise that yet smaller vessels can be cannulated, with resulting greater selectivity for target organ damage. At present, conventional methods are likely to be successful in most patients and those remaining are unlikely to benefit significantly from alcohol ablation.

A D CUNNINGHAM

Royal Brompton National Heart and Lung Hospital, E ROWLAND

Sydney Street,

London $S W 36 N P$

1 Evans GT, Scheinman MM, Benditt D, et al. The percutaneous cardiac mapping and ablation registry: final summary of results. $P A C E$ 1988;11:1621-6.

2 Evans GT, Huang WH, and the CAR Investigators. Comparison of direct current and radiofrequency energy for catheter ablation of the atrioventricular junction: results of a prospective multicenter study [Abstract]. Circulation 1990;82 (suppl III):719.

3 Rowland E, Cunningham D, Ahsan A, Rickards A. Transvenous ablation of atrioventricular conduction with a low energy power source. Br Heart 1989;62:361-6.

4 Ahsan AJ, Cunningham AD, Rickards AF, Rowland E. Low energy ablation of atrioventricular conduction [Abstract]. Eur Heart $J$ 1990;11 (abstract suppl): 233 .

5 Lemery R, Talajic M, Roy D, Girard A, Montpetit M. Low energy direct current ablation in patients with supraventricular tachycardia [Abstract]. Am Coll Cardiol 1990;15:20A.

6 Schläpfer J, Fromer M, Goy JJ, Kappenberger L. Modulation of A-V node conduction for supraventricular tachyarrhythmias: comparison of 2 techconduction for supraventricular tachyarrhythmias: compariso
niques [Abstract]. Eur Heart $J$ 1990;11 (abstract suppl): 164 .

7 Lavergne TL, Sebag CI, Le Heuzey J-Y, et al. Closed-chest radio-frequency avergne TL, Sebag CI, Le Heuzey J-Y, et al. Closed-chest radio-frequency
modification of atrioventricular conduction using a suction catheter: long modification of atrioventricular conduction using a suction
term results [Abstract). Circulation 1990;82 (suppl IIII):719.

8 Morady F, Kadish A, Calkins $\mathrm{H}$, et al. Diagnosis and immediate cure of paroxysmal supraventricular tachycardia [Abstract]. Circulation 1990;82 (suppl III):689. 